\title{
Stretching of Jordan Arc Contact Systems
}

\author{
Hubert de Fraysseix and Patrice Ossona de Mendez \\ UMR 8557, CNRS, Paris, France
}

\begin{abstract}
We prove that a contact system of Jordan arcs is stretchable if and only if it is extendable into a weak arrangement of pseudo-lines.
\end{abstract}

\section{Barycentric Representations of Graphs}

\subsection{Tutte's Barycenter Theorem}

It is a classical result independently established by Wagner [11], Fáry [2] and Stein [6] (which is also a consequence of the Steinitz's theorem on convex polytopes [7]) that any simple planar graph has a straight line representation in the plane. In the early 60's, Tutte 9] 10] gave a way to build a convex embedding of a 3-connected planar graph:

- Let $S$ be the vertex set of a face $F_{0}$ of $G$ in a planar embedding of $G$,

- let $f: S \rightarrow \mathbb{R}^{2}$ be a mapping of the vertices in $S$ to the points of a convex polygon, in such a way that the order of the points on the polygon is the same as the order of the vertices on the face $F_{0}$,

- let $\Psi: V(G) \backslash S \rightarrow \mathbb{R}^{2}$ be the solution of the linear system

$$
\forall x \in V(G) \backslash S, \quad \Psi(x)=\sum_{\{v, x\} \in E(G)} \frac{1}{d(x)} \Psi(v)
$$

Theorem 1 (Tutte's theorem). $\Psi$ defines an embedding of $G$ into the plane, with strictly convex interior faces.

In his quite long and difficult proof, Tutte simultaneously reproves Kuratowski's planarity criterion. Other proofs of this theorem have been published since. This result has been generalized by Thomassen to infinite planar graphs in [8], while Linial, Lovász and Wigderson proved the equivalence of the $k$ vertex connectivity and the existence of a convex embedding (for any $k$ extremal vertices) in general position in $\mathbb{R}^{k}[3$. In Tutte's construction, each vertex in $V(G) \backslash S$ is the barycenter of its neighbors. Although Tutte assumed all the coefficients to be equal, his proof extends without changes when coefficients are positive reals. In the aim of studying straight line segments contact systems, we shall first give a short proof of an extension of this theorem, in a framework allowing null coefficients, giving some side results necessary for our study on stretchability. 
First, we shall give necessary and sufficient conditions for such a barycentric linear system to have a unique solution (without planarity assumptions), and for its solution to define a plane embedding (assuming that the graph is planar and that the source set is embedded on a convex polygon with a compatible point order). Instead of the classic approach consisting in directly proving the planarity of the barycentric representation, we will make use of continuity arguments, while considering an homotopy from some initial straight line embedding of a triangulation of the graph (see also [1] [4] for alternative approaches and generalizations of Tutte's Theorem).

\subsection{Definitions and Notations}

A barycentric system $\Sigma$ on a graph $G$ is a triple $(S, f, \alpha)$, where the source set $S$ of $\Sigma$ is a subset of vertices of $G$, the limit function $f$ of $\Sigma$ is a function from $S$ to $\mathbb{R}^{k}$, the weight function $\alpha$ of $\Sigma$ is a function from $V(G) \times V(G)$ to $[0,1]$, and such that:

$$
\begin{gathered}
\forall x, y \in V(G), \quad \alpha(x, y) \neq 0 \Rightarrow\{x, y\} \in E(G) \text { and } x \notin S \\
\forall x \in V(G) \backslash S, \quad \sum_{(x, v) \in E(G)} \alpha(x, v)=1
\end{gathered}
$$

A solution of $\Sigma$ is a function $\Psi: V(G) \rightarrow \mathbb{R}^{k}$, such that:

$$
\Psi(x)= \begin{cases}f(x), & \text { if } x \in S \\ \sum_{v \in V(G)} \alpha(x, v) \Psi(v), & \text { otherwise }\end{cases}
$$

Remark 1. Notice the following equivalences:

$$
x \in S \quad \sum_{(x, v) \in E(G)} \alpha(x, v)=0 \quad \Longleftrightarrow \quad \forall v \in V(G), \quad \alpha(x, v)=0
$$

Let $\Sigma=(S, f, \alpha)$ be a barycentric system on a simple graph $G$. Let $A$ be a subset of $V(G)$, the relative source set $S(A)$ of $A$ is defined by:

$$
S(A)=(S \cap A) \cup\{x \in A, \exists v \notin A, \quad \alpha(x, v) \neq 0\}
$$

That is, $\forall x \in A$ :

$$
x \notin S(A) \quad \Longleftrightarrow \quad \sum_{(x, v) \in E\left(G_{A}\right)} \alpha(x, v)=1
$$

Notice that, according to this definition, $S(V(G))=S$.

Lemma 1. Let $G$ be a simple graph, let $\Sigma=(S, f, \alpha)$ be a barycentric system on $G$. Assume $\Psi$ is a solution of $\Sigma$. 
Let $A$ be a subset of $V(G)$. We define $\alpha_{A}: A \times A \rightarrow[0,1]$ by:

$$
\forall x, y \in A, \quad \alpha_{A}(x, y)= \begin{cases}0, & \text { if } x \in S(A) \\ \alpha(x, y), & \text { otherwise }\end{cases}
$$

Then, $\Sigma_{A}=\left(S(A), \Psi_{\mid S(A)}, \alpha_{A}\right)$ is a barycentric system on $G_{A}$, having $\Psi_{\mid A}$ as a solution.

Proof. According to the definition of $S(A)$, the function $\alpha_{A}$ is such that:

$$
\begin{aligned}
\forall x, y \in A, & \alpha(x, y) \neq 0 & \Rightarrow\{x, y\} \in E\left(G_{A}\right) \text { and } x \notin S(A) \\
\forall x \in A \backslash S(A), & \sum_{(x, v) \in E\left(G_{A}\right)} \alpha(x, v) & =1
\end{aligned}
$$

Thus, $\Sigma_{A}$ is a barycentric system on $G_{A}$, obviously having $\Psi_{\mid A}$ as a solution.

A barycentric system $\Sigma=(S, f, \alpha)$ on a simple graph $G$ defines a directed graph $D_{\Sigma}$ on the same vertex set as $G$, having $S$ has its set of sources:

$$
\forall x, y \in V(G), \quad(x, y) \in E\left(D_{\Sigma}\right) \Longleftrightarrow \alpha(y, x) \neq 0
$$

Let $G$ be a simple graph of order $n$. Consider any fixed numbering of the vertices of $G$. A barycentric system $\Sigma=(S, f, \alpha)$ on $G$ define a $n \times n$ square matrix $A$ (with coefficients in $[0,1]$ ) and a $n$ column matrix $F$ (with coefficients in $\left.\mathbb{R}^{k}\right)$ by $A_{i, j}=\alpha\left(v_{i}, v_{j}\right)$ and

$$
F_{i}= \begin{cases}f\left(v_{i}\right) & \text { if } v_{i} \in S \\ \mathbf{0} & \text { otherwise }\end{cases}
$$

So, the solutions of $\Sigma$ correspond to the solutions $\Psi$ of the equation $\Psi=A \Psi+F$.

Let $G$ be a simple graph, $\Sigma=(S, f, \alpha)$ a barycentric system on $G$, and $D_{\Sigma}$ the associated directed graph.

The distance function $\operatorname{dist}_{S}: V(G) \rightarrow \mathbb{N} \cup\{\infty\}$ is defined as the minimum length of a directed path in $D_{\Sigma}$ from $S$ to a vertex, or $\infty$ is such a path does not exist.

\subsection{General Resolution}

In the next lemmas, $G$ is assumed to be a simple graph, and $\Sigma=(S, f, \alpha)$ to be barycentric system on $G$. Elements of $\mathbb{R}^{k}$ are compared with respect to lexicographic order.

Lemma 2. If $\sup _{v \in V(G)} \operatorname{dist}_{S}(v)<\infty$, any barycentric function $\Psi$ reaches its extremal values on $S$ and, for any vertex $x$, there exists in $D_{\Sigma}$ :

- a directed path $P_{x}^{+}$from $S$ to $x$ such that $\left(\Psi\right.$, dist $\left._{S}\right)$ is strictly increasing on $P_{x}^{+}$(with respect to lexicographic order). 
- a directed path $P_{x}^{-}$from $S$ to $x$ such that $\left(\Psi,-\operatorname{dist}_{S}\right)$ is strictly decreasing on $P_{x}^{-}$(with respect to lexicographic order).

Proof. We only have to prove the existence of $P_{x}^{+}$as the existence of the directed path $P_{x}^{-}$is then obtained by considering $-\Psi$ instead of $\Psi$, and the monotony of these paths implies that the extrema of $\Psi$ are reached on $S$.

Assume there is at least a vertex $v$ such that such a path $P_{v}^{+}$does not exist, and choose the one with the smallest possible value of $\left(\Psi(v), \operatorname{dist}_{S}(v)\right)$. Obviously, $v$ does not belong to $S$. So,

$$
\Psi(v)=\sum_{(w, v) \in E\left(D_{\Sigma}\right)} \alpha(v, w) \Psi(w) \geq \min _{(w, v) \in E\left(D_{\Sigma}\right)} \Psi(w)
$$

- If there exists an $\operatorname{arc}(x, v) \in E\left(D_{\Sigma}\right)$ such that $\Psi(x)<\Psi(v)$, then $P_{x}^{+}$exists (by minimality assumption on $v$ ) and the directed path $P_{v}^{+}=P_{x}^{+}+(x, v)$ contradicts the hypothesis.

- Otherwise, any edge $(x, v) \in E\left(D_{\Sigma}\right)$ is such that $\Psi(x)=\Psi(v)$. As there exists an edge $(x, v) \in E\left(D_{\Sigma}\right)$, such that $\operatorname{dist}_{S}(x)=\operatorname{dist}_{S}(v)-1$ the directed path $P_{x}^{+}$exists, and $P_{v}^{+}=P_{x}^{+}+(x, v)$ contradicts the hypothesis.

In both cases, we are led to a contradiction.

Lemma 3. If $\sup _{v \in V(G)} \operatorname{dist}_{S}(v)<\infty, \Sigma$ has a unique solution $\phi$.

Proof. The function $\phi$ exists and is unique if and only if the kernel of $A$ is $\{0\}$, that is, if and only if the barycentric system $\left(S, f_{0}, \alpha\right)$ has no non zero solution, where $f_{0}$ is a constant function mapping $S$ to $\mathbf{0}$. But this is an implication of the previous result, which asserts that the extrema of a solution of a barycentric system are reached on its source set, that is that a solution has the same extremal values as the limit function.

Lemma 4. If $\sup _{v \in V(G)} \operatorname{dist}_{S}(v)=\infty$, the barycentric system has an infinite set of solutions.

Proof. Let $V_{S}$ be the subset of vertices reachable from $S$ by a directed path. For any value $c \in \mathbb{R}^{k}$, define the function $f^{\prime}:\left(V(G) \backslash V_{S}\right) \cup S \rightarrow \mathbb{R}^{k}$ by $f^{\prime}(v)=f(v)$ if $v \in S$, and $f^{\prime}(v)=c$, otherwise. According to Lemma 3 the barycentric system $\left(\left(V(G) \backslash V_{S}\right) \cup S, \alpha, f^{\prime}\right)$ has a solution $\phi$, which is also a solution of $\Sigma$. This solution is such that $\left.\phi\right|_{V(G) \backslash V_{S}} \equiv c$, and thus we get a infinite set of solutions of $\Sigma$.

Theorem 2. Let $G$ be a simple graph. Any barycentric system $\Sigma=(S, f, \alpha)$ has a solution, which is unique if and only if any vertex of the directed graph $D_{\Sigma}$ associated with $\Sigma$ may be reached from $S$ by a directed path.

In the latter case, the solution $\phi$ reaches its extremal values on $S$ and, for any vertex $x$, there exists in $D_{\Sigma}$ : 
- a directed path $P_{x}^{+}$from $S$ to $x$ such that $\left(\phi\right.$, dist $\left._{S}\right)$ is strictly increasing on $P_{x}^{+}$(with respect to lexicographic order).

- a directed path $P_{x}^{-}$from $S$ to $x$ such that $\left(\phi,-\right.$ dist $\left._{S}\right)$ is strictly decreasing on $P_{x}^{-}$(with respect to lexicographic order).

Proof. This is a direct consequence of the three preceding propositions.

Corollary 1. Let $G$ be a simple graph and let $\Sigma=(S, f, \alpha)$ be a barycentric system on $G$ having a unique solution.

Then, for any subset $A$ of $V(G),|S(A)|$ is at least equal to the number of connected components of $G_{A}$.

Proof. It is sufficient to prove the case where $G_{A}$ is connected. Let $x$ be an element of $A$. If $x$ belongs to $S$, we are done. Otherwise, as $\Sigma$ has a unique solution, there exists, according to Theorem 2, a directed path $P$ from $S$ to $x$. This path enters $A$ at vertex $v$ which, by definition, belongs to $S(A)$.

\subsection{Planarity of a Barycentric Representation}

Barycentric representations and convex drawings. Let $G$ be a 2connected planar graph. A straight line drawing of $G$ is a convex drawing if each face of the graph is drawn as a convex polygon. The drawing is strictly convex if the vertices of a face are mapped to the extremal points of the polygon corresponding to the face. A barycentric representation of $G$ with source set $S \subseteq V(G)$ is a mapping from $V(G)$ to $\mathbb{R}^{2}$, defining a straight line planar drawing of $G$, and such that any vertex not in $S$ is a barycenter of its neighbors (with coefficients in $[0,1]$ ). Convex drawings are deeply related to barycentric representations:

Lemma 5. Let $G$ be a 2-connected planar graph. A straight line representation of $G$ is convex if and only it is a barycentric representation of $G$, which source set $S$ is the vertex set of a face of $G$ and is embedded on a convex polygon (in a compatible order).

Proof. Consider a convex drawing of $G$ and let $S$ be the vertex set of the external face. Let $v$ be an internal vertex. As the faces including $v$ are convex, we may augment the neighborhood of $v$ to a wheel by adding straight line segments between consecutive non-adjacent neighbors while preserving planarity. Thus, $v$ belongs to the convex hull of its neighbors. Hence, the drawing is a barycentric representation of $G$ with source set $S$.

Conversely, assume $S$ is the vertex set of a face of $G$ embedded on a convex polygon of the plane. Assume the representation includes a non convex internal face, and let $v_{1}, v_{2}, v_{3}$ be three consecutive vertices of such a face defining a concave angle at $v_{2}$. Then, $v_{2}$ does not belong to the convex hull of its neighbors, a contradiction. 
Corollary 2. The embedding defined by a convex drawing of a 2-connected planar graph $G$ is uniquely determined by the choice of the external face.

Corollary 3. Let $P$ be a convex polygon, let $\left(p_{1}, p_{2}, p_{3}\right)$ be a triangle including $P$ in its interior, let $G$ be the graph induced by $P$, the triangle and a triangulation of the annulus between the triangle and the polygon, let $s_{1}, s_{2}, s_{3}$ be the vertices of $G$ mapped to $p_{1}, p_{2}, p_{3}$ and let $g\left(s_{i}\right)=p_{i}($ for $1 \leq i \leq 3)$.

Then, there exists a function $\alpha_{P}: V(G) \times V(G) \rightarrow[0,1]$, such that $\Sigma=$ $\left(\left\{s_{1}, s_{2}, s_{3}\right\}, g, \alpha_{P}\right)$ is a barycentric system on $G$ with unique solution $\Psi$ mapping $G$ to its geometric embedding. A simple geometric argument shows that $\alpha$ may be chosen in such a way that $\alpha(x, y)>0$, for any pair $(x, y)$ of points adjacent on $P$.

\section{Barycentric Representations}

Lemma 6. Let $G$ be a maximal planar graph and let $\mu:[0,1] \times V(G) \rightarrow \mathbb{R}^{2}$ be a mapping such that

- $\mu(0, \cdot)$ induces a plane straight line representation of $G$,

$-\mu(\cdot, x)$ is continuous for any vertex $x$ of $G$,

- $\forall t \in] 0,1]$ and for all triangle $\left(x_{1}, x_{2}, x_{3}\right)$ of $G, \mu\left(t, x_{1}\right), \mu\left(t, x_{2}\right)$ and $\mu\left(t, x_{3}\right)$ are not collinear.

Then, $\mu(t, \cdot)$ induces a plane straight line representation of $G$ for every $t$ in $[0,1]$.

Proof. In order to check if a straight line representation of a maximal planar graph is plane, it is sufficient to check that no edge has null length, that no two adjacent edges are overlapping and that the circular orders of the edges around a vertex define a planar map. The first two cases may not occur, as it would imply the existence of an aligned triangle. As $\mu(\cdot, x)$ is continuous for any vertex $x$, the circular order around a vertex may only change if two consecutive edges overlap for some $t$, which would give rise to a contradiction.

Given a weak plane straight line representation of $G$, a connected subgraph $H$ is 0 -degenerated if all its vertices are mapped to a same point. It is 1-degenerated if all its vertices are mapped to aligned points. A $k$-degenerated component is a maximal $k$-degenerated connected subgraph (it is thus an induced subgraph of $G)$.

Lemma 7. Let $H$ be a 2-connected plane graph, and let $\Sigma=\left(S_{H}, f_{H}, \alpha_{H}\right)$ be a barycentric system on $H$ having a unique solution $\Psi_{H}$, where $S_{H}$ is the vertex set of the outer face of $H$.

Assume $f_{H}$ maps $S_{H}$ to a convex polygon $P$ in compatible order, and that the following condition holds for any subset $A$ of vertices inducing a connected subgraph:

(i) $|S(A)|=1 \Rightarrow|A|=1$ 
(ii) $|S(A)|=2 \Rightarrow H_{A}$ is a path

Then, there exists a maximal planar graph $G$ and a barycentric system $\Sigma=$ $(S, f, \alpha)$ having a unique solution, such that:

- $H$ is a subgraph of $G$,

- $\Psi_{\mid V(H)}$ is the solution of $\Sigma_{H}$,

- conditions (i) and (ii) hold for $G$ (replacing $H_{A}$ by $G_{A}$, and considering relative source sets according to $\Sigma$ )

Proof. Denote $S^{(H)}$ and $S^{(G)}$ the relative source set functions with respect to $H, \Sigma_{H}$ and $G, \Sigma_{G}$, respectively.

The graph $G_{1}$ is obtained by adding a vertex in each interior face of $H$, adjacent to all the vertices of the face.

Let $\left\{p_{1}, p_{2}, p_{3}\right\}$ be a triangle having the polygon $P$ in its interior, let $G_{2}$ be the graph obtained by geometrically triangulating the annulus between the triangle and the polygon, let $s_{1}, s_{2}, s_{3}$ be the vertices of $G_{2}$ corresponding to the $p_{1}, p_{2}, p_{3}$, let $f$ be the mapping defined by $f\left(s_{i}\right)=p_{i}$, and let $\alpha_{P}$ be such that $\left(\left\{s_{1}, s_{2}, s_{3}\right\}, f, \alpha_{P}\right)$ is a barycentric system on $G_{2}$ having a unique solution $\Psi$, which restriction to $S$ is $f_{H}$ (such a function exists according to Corollary 3).

The graph $G$ is obtained from the union of $G_{1}$ and $G_{2}$, by identifying the vertices corresponding to elements of $S$.

Then, define $\alpha: V(G) \times V(G) \rightarrow[0,1]$ as follows:

$$
\alpha(x, y)= \begin{cases}\alpha_{H}(x, y), & \text { if } x, y \in V(H) \\ \alpha_{P}(x, y), & \text { if } x, y \in V\left(G_{2}\right) \\ \frac{1}{d(x)}, & \text { if } x \in V\left(G_{1}\right) \backslash V(H) \text { and } y \in V(H), \\ 0, & \text { otherwise }\end{cases}
$$

By construction, $H$ is a subgraph of $G$. It is easily checked that $\Sigma=$ $\left(\left\{s_{1}, s_{2}, s_{3}\right\}, f, \alpha\right)$ is a barycentric system on $G$ having a unique solution, which restriction to $V(H)$ is a solution of $\Sigma_{H}$ (according to Lemma 1).

Consider any subset $A \subseteq V(G)$ inducing a connected subgraph $G_{A}$ of $G$, such that $\left|S_{G}(A)\right| \leq 2$ and $H_{A}$ has no cycle. As no vertex $x \in A \backslash V(H) \backslash\left\{s_{1}, s_{2}, s_{3}\right\}$ may have all its neighbors in $A$ (for otherwise its neighbors would form a cycle in $H_{A}$ ), any vertex in $A \backslash V(H)$ belongs to $S^{(G)}(A)$. By definition, for any $x \in S^{(H)}(A \cap V(H)) \backslash S_{H}$, there exists $v \in V(H) \backslash A, \alpha_{H}(x, v)>0$. Thus, $\alpha(x, v)>0$, what implies $x \in S^{(G)}(A)$. If $S^{(H)}(A) \cap S_{H}$ is not empty, it is easily checked that either $S^{(H)}(A) \cap S_{H}$ is included in $S^{(G)}(A)$, or $S_{H}$ is included in $A$ (and hence $H_{A}$ includes a cycle), or $S^{(H)}(A)$ has cardinality at least 3 . Altogether, we get:

$$
S^{(G)}(A)=S^{(H)}(A \cap V(H)) \cup(A \backslash V(H))
$$

Assume there exists a subset $A$ of $V(G)$ inducing a connected subgraph of $G$. 
If $\left|S^{(G)}(A)\right|=1$ then, according to (9), either $A \subseteq V(H)$ and $\left|S^{(H)}(A)\right|=$ 1, or $A \cap V(H)=\emptyset$ and $|A|=1$. Hence, (i) holds.

If $\left|S^{(G)}(A)\right|=2$ then, according to (9):

- either $A \subseteq V(H)$ and $\left|S^{(H)}(A)\right|=2$, and hence $G_{A}=H_{A}$ is a path,

- or $A \backslash V(H)=1$ and $\left|S^{(H)}(A)\right|=1$, and hence $|A|=2$ and $G_{A}$ is a path,

- or $A \cap V(H)=\emptyset$ and $A$ is a subset of size 2 of $\left\{s_{1}, s_{2}, s_{3}\right\}$ (hence inducing a path).

Thus (ii) holds for $G$.

Theorem 3. Let $G$ be a 2-connected plane graph, $S$ the vertex set of its outer face, $f$ a function mapping $S$ to the points of a convex polygon in compatible order, and $\alpha: V(G) \times V(G) \rightarrow[0,1]$, such that $\Sigma=(S, f, \alpha)$ is a barycentric system on $G$ with a unique solution $\Psi$.

Then, $\Psi$ defines a barycentric representation of $G$ if and only if, for any subset $A$ of $V(G)$ inducing a connected subgraph, the following assertions hold:

(i) $|S(A)|=1 \Rightarrow|A|=1$

(ii) $|S(A)|=2 \Rightarrow G_{A}$ is a path

Proof. First, notice that the necessity of conditions (i) and (ii) is straightforward.

Assume (i) and (ii) hold. According to Lemma 7, we may assume that $G$ is maximal planar and that $f$ maps its outer face $\left\{s_{1}, s_{2}, s_{3}\right\}$ to a triangle $\left\{p_{1}, p_{2}, p_{3}\right\}$. It is a classical result [2,611] that $G$ has a straight line embedding $\Gamma$ with external face $\left\{s_{1}, s_{2}, s_{3}\right\}$. Moreover, using an affine transformation, we may assume that $s_{1}, s_{2}, s_{3}$ are mapped to $p_{1}, p_{2}, p_{3}$ in this embedding. According to Lemma 5, this is a barycentric representation, and thus induced by the solution of some barycentric system $\Sigma_{x}^{\prime}=\left(S, f, \alpha^{\prime}\right)$. Let $\alpha_{t}=(1-t) \alpha^{\prime}+\alpha$. Then, $\Sigma^{(t)}=\left(S, f, \alpha_{t}\right)$ is obviously a barycentric system having a unique solution $\Psi^{(t)}$ continuously depending on $t$. Let $\mu(t, v)=\Psi^{(t)}(v)$. If, for any $\left.\left.t \in\right] 0,1\right]$, there exists no triangle $\left(x_{1}, x_{2}, x_{3}\right)$ such that $\mu\left(t, x_{1}\right), \mu\left(t, x_{2}\right)$ and $\mu\left(t, x_{3}\right)$ are collinear then, according to Lemma 6, $\Psi=\mu(1, \cdot)$ will define a barycentric representation of $G$. First notice that, if $0<t<1$ then, for any $x, y \in V(G), \alpha_{t}(x, y)>0$ if and only if $\alpha(x, y)>0$ or $\alpha^{\prime}(x, y)>0$. Thus, for any subset $A$ of vertices, the relative source set of $A$ computed according to $\Sigma_{t}$ includes the relative source set of $S(A)$ computed according to $\Sigma$. Hence, as (i) and (ii) hold for $t=1$, these conditions also hold for any $t \in] 0,1]$. Moreover, if conditions (i) and (ii) imply that no triangle $\left(x_{1}, x_{2}, x_{3}\right)$ of $G$ is such that $\mu\left(1, x_{1}\right), \mu\left(1, x_{2}\right)$ and $\mu\left(1, x_{3}\right)$ are collinear then the same will apply for any $t \in] 0,1[$.

Assume there exists a triangle $\left\{x_{1}, x_{2}, x_{3}\right\}$ of $G$, such that $\Psi\left(x_{1}\right), \Psi\left(x_{2}\right)$ and $\Psi\left(x_{3}\right)$ are collinear. Let $G_{A} \subseteq\left\{x_{1}, x_{2}, x_{3}\right\}$ be the corresponding 1-degenerated component, and let $a x+b y+c=0$ be an equation of the straight line $\Delta$ including $\Psi(A)$. Consider the barycentric system $\Sigma_{\Delta}=\left(S, a f_{x}+b f_{y}+c, \alpha\right)$ (where $\left.\left(f_{x}(v), f_{y}(v)\right)=f(v)\right)$ and its unique solution $\Psi$. The subgraph $G_{A}$ is then a connected component of $\Psi^{-1}(0)$. 
Let $G^{+}$be the directed planar graph obtained from $G$ by adding to vertices $\omega^{-}$and $\omega^{+}$and the edges $\left\{\omega^{-}, s\right\}$ (if $s \in S$ and $\phi(s) \leq 0$ ), $\left\{\omega^{+}, s\right\}$ (if $s \in$ $S$ and $\phi(s) \geq 0)$ and $\left\{\omega^{-}, \omega^{+}\right\}$.

For each vertex $a_{i} \in S(A) \backslash S$, there exists a vertex $v$ such that $\left(v, a_{i}\right)$ is an arc of $D_{\Sigma_{\Delta}}$ and $\Psi(v) \neq 0$. From the equation $0=\Psi\left(a_{i}\right)=\sum_{\left(x, a_{i}\right) \in E\left(D_{\Sigma_{\Delta}}\right)} \Psi(x)$, we deduce that there exists, for each such $a_{i}$, two vertices $x_{i}$ and $y_{i}$, such that $\left(x_{i}, a_{i}\right)$ and $\left(y_{i}, a_{i}\right)$ are arcs of $D_{\Sigma_{\Delta}}$ and such that $\Psi\left(x_{i}\right)<0<\Psi\left(y_{i}\right)$. According to Theorem 2, there exists in $D_{\Sigma_{\Delta}}$, for each such $a_{i}$, directed paths $P_{a_{i}}^{+}$and $P_{a_{i}}^{-}$, whose vertices (except $a_{i}$ ) have a negative (resp. positive) $\Psi$-value.

Thus, there exists, in $G^{+}$, for each $a_{i} \in S(A)$, paths $P_{1}\left(a_{i}\right)$ and $P_{2}\left(a_{i}\right)$, from $\omega^{-}$(resp. $\left.\omega^{+}\right)$to $a_{i}$, which vertices (except $a_{i}$ ) have a negative (resp. positive) $\Psi$-value. Hence, we have:

$$
\begin{aligned}
\forall\left\{a, a^{\prime}\right\} \subseteq S(A) & P_{1}(a) \cap P_{2}\left(a^{\prime}\right)=\emptyset \\
\forall a & \in S(A), \quad P_{1}(a) \cap A=P_{2}(a) \cap A=P_{1}(a) \cap P_{2}(a)=\{a\} \\
\forall a & \in S(A), \quad \omega^{-} \in P_{1}(a) \text { and } \omega^{+} \in P_{2}(a)
\end{aligned}
$$

Hence, two cases may occur:

- $A=S(A)$. Then, the triangle $\left\{x_{1}, x_{2}, x_{3}\right\}$, the paths $P_{i}\left(x_{j}\right)(i \in\{1,2\}, j \in$ $\{1,2,3\})$, and the edge $\left\{\omega^{-}, \omega^{+}\right\}$may be contracted to $K_{5}$, contradicting the planarity of $G^{+}$.

- there exists a vertex $x$ in $A \backslash S(A)$. According to the 3-connexity of $G^{+}$, there exists 3 disjoint paths from $x$ to $\omega^{+}$. By continuity, these paths leave $A$ at some distinct vertices $v_{1}, v_{2}, v_{3} \in S(A)$. Then, the subpaths from $x$ to $v_{1}, v_{2}, v_{3}$, and the paths $P_{i}\left(v_{j}\right)(i \in\{1,2\}, j \in\{1,2,3\})$ may be contracted to a $K_{3,3}$, contradicting the planarity of $G^{+}$.

In both cases, we are led to a contradiction. Thus, no triangle of $G$ may be mapped to 3 collinear points. Hence, $\Psi$ is a barycentric representation of $G$.

\section{Jordan Arc Contact Systems}

\subsection{Introduction}

A Jordan arc is an arc homeomorphic to a straight line segment. A pseudo-line is an arc homeomorphic to a straight line. A contact system is a set of Jordan arcs in the plane, such that two arcs intersect at most once at a point which is internal to at most one arc. A contact system is stretchable if there exits an homeomorphism which transforms it into a contact system whose arcs are straight line segments.

A set $\mathcal{L}=\left(L_{1}, \ldots, L_{k}\right)$ of pseudo-lines is a weak arrangement of pseudo-lines if it satisfies :

$$
\forall i \neq j, \quad\left|L_{i} \cap L_{j}\right| \leq 1
$$


A weak arrangement of pseudolines is an arrangement of pseudolines if it satisfies the additional axiom :

$$
\forall i, j, k, \quad\left(L_{i} \cap L_{j}=\emptyset \text { and } L_{i} \cap L_{k} \neq \emptyset\right) \Rightarrow L_{j} \cap L_{k} \neq \emptyset
$$

A contact system $\mathcal{A}=\left(A_{1}, \ldots, A_{k}\right)$ is extendable into an arrangement of pseudo-lines (resp. into a weak arrangement of pseudolines) if there exists a arrangement of pseudo-lines (resp. a weak arrangement of pseudolines) $\mathcal{L}=$ $\left(L_{1}, \ldots, L_{k}\right)$, such that $A_{i} \subseteq L_{i}$ for each $i \in\{1, \ldots, k\}$.

Remark 2. It is easy to prove that a contact system is extendable into an arrangement of pseudo-lines if and only if it is extendable into a weak arrangement of pseudo-lines.

\subsection{Extendibility and Extremal Points}

An extremal point of a contact system is a point of the union of the arcs which is interior to no arc. We note $\delta(\mathcal{A})$ the set of the extremal points of the contact system $\mathcal{A}$. A maximal contact system is a contact system whose extremal points belong to the unbounded region. A contact system $\mathcal{A}=\left(A_{1}, \ldots, A_{k}\right)$ is extendable into a maximal contact system if there exists a maximal contact system $\mathcal{A}^{\prime}=\left(A_{1}^{\prime}, \ldots, A_{k}^{\prime}\right)$, such that $A_{i} \subseteq A_{i}^{\prime}$ for each $i \in\{1, \ldots, k\}$.

Remark 3. If a contact system is extendable into a weak arrangement of pseudolines, it is extendable into a maximal contact system, which in turn is extendable into a weak arrangement of pseudo-lines.

Let $\mathcal{A}$ be a maximal contact system extendable into a weak arrangement of pseudo-lines $\mathcal{L}$.

- Let $p$ be an interior point of an arc $A$ (but no other arc) on the unbounded region of $\mathcal{A}$.

- Let $L$ be the pseudoline extending $A$ in $\mathcal{L}$, and let $L^{-}$and $L^{+}$be the two halves of $L$ delimited by $p$.

- Let $H$ be a half pseudo-line having its endpoint at $p$ and having no other intersection in $\mathcal{A}$.

Then, $L$ and $H$ induce a "partition" of $A$ into three new contact systems $A^{-}, A^{+}$ and $A^{0}$ (see Fig. 1):

- $H, L^{-}$and $L^{+}$define three unbounded regions $\mathcal{R}^{-}, \mathcal{R}^{+}$and $\mathcal{R}^{0}$ having $H \cup$ $L^{-}, H \cup L^{+}$and $L$ as respective frontiers.

- We define $J^{-}, J^{+}$and $J^{0}$ as the sub-arcs of $H \cup L^{-}, H \cup L^{+}$and $L$ strictly including all the intersections of these later pseudolines with $\mathcal{A}$.

These regions define three contact systems of Jordan arcs :

$$
\begin{array}{ll}
\mathcal{A}^{-}=\left\{J^{-}\right\} \cup\left\{A \cap \mathcal{R}^{-},\right. & \left.A \in \mathcal{A} \text { and } A \cap \mathcal{R}^{-} \text {is a nonempty arc }\right\} \\
\mathcal{A}^{+}=\left\{J^{+}\right\} \cup\left\{A \cap \mathcal{R}^{+},\right. & \left.A \in \mathcal{A} \text { and } A \cap \mathcal{R}^{+} \text {is a nonempty arc }\right\}
\end{array}
$$





Fig. 1. Partitioning a Contact System

$\mathcal{A}^{0}=\left\{J^{0}\right\} \cup\left\{A \cap \mathcal{R}^{0}, \quad A \in \mathcal{A}\right.$ and $A \cap \mathcal{R}^{0}$ is a nonempty arc $\}$

These three contact systems have some nice properties :

Lemma 8. The contact systems $\mathcal{A}^{-}, \mathcal{A}^{+}$and $\mathcal{A}^{0}$ are maximal and extendable into weak arrangements of pseudo-lines.

Proof. The maximality is straightforward, as the extremal points of these contact systems are either extremal points of $\mathcal{A}$ or the extremities of $J^{-}, J^{+}$or $J^{0}$.

The contact systems $\mathcal{A}^{-}$and $\mathcal{A}^{+}$are homeomorph to the contact systems where $J^{-}$(resp. $J^{+}$) is replaced by $J^{0}$. Then, the weak arrangement of pseudolines which extends $\mathcal{A}$ is an extension of all of the contact systems.

Lemma 9. If the arc $A$ includes no extremal point of $\mathcal{A}$, we have :

$$
2 \leq\left|\mathcal{A}^{-}\right|<|\mathcal{A}|, \quad 2 \leq\left|\mathcal{A}^{+}\right|<|\mathcal{A}|, \quad 3 \leq\left|\mathcal{A}^{0}\right| \leq|\mathcal{A}|,
$$

and each of $\mathcal{A}^{-}, \mathcal{A}^{+}$and $\mathcal{A}^{0}$ has two extremal points belonging to a same arc.

Proof. As $A$ includes no extremal point of $\mathcal{A}$ the extremity of $A$ in $A^{-}$(resp. $\left.A^{+}\right)$is interior to some arc $B^{-}$(resp. $B^{+}$). As $A$ may not intersect an arc twice, 
we have $B^{-} \neq B^{+}$. Moreover, each of these arcs intersects at most once the extension $L$ of $A$, and hence the arc $B^{-}$(resp. $B^{+}$) does not meet the region $\mathcal{R}^{+}$ (resp. $\mathcal{R}^{-}$). Thus, $\left|\mathcal{A}^{+}\right| \leq|\mathcal{A}|-1$ and the same holds for $\left|\mathcal{A}^{-}\right|$. Notice also that $\mathcal{A}^{-}$and $\mathcal{A}^{+}$include at least two arcs : $J^{-}$(resp. $J^{+}$) with two extremal points on it and the non empty portion of $B^{-}\left(\operatorname{resp} . B^{+}\right)$in $\mathcal{R}^{-}\left(\operatorname{resp} . \mathcal{R}^{+}\right)$. Moreover, $\mathcal{A}^{0}$ includes at least three arcs : $J^{0}$ (with two extremal points on it) and the portions of $B^{-}$and $B^{+}$in $\mathcal{R}^{0}$.

Lemma 10. Any contact system extendable into an arrangement of pseudolines has at least 3 extremal points, unless it has cardinality at most one.

Proof. First notice that we may prove the lemma for maximal contact systems only, according Remark 3 and the fact that the extended contact system may not have more extremal points than the original one.

The lemma is straightforward for contact systems of cardinality at most two. So, assume that for any $2 \leq i<k$, the lemma holds for maximal contact systems of cardinality $i$ and assume there exists a maximal contact system $\mathcal{A}$ of cardinality $k$ having at most 2 extremal points.

Notice that at least $3 \operatorname{arcs}$ of $\mathcal{A}$ meet the unbounded region in a sub-arc. Otherwise, the contact system would be bounded by a single arc or by two arcs intersecting each other twice, and the system could not be extended into a weak arrangement of pseudo-lines.

- Assume $\mathcal{A}$ has two extremal points belonging to a same arc.

From the previous construction and according to Lemma $8, \mathcal{A}^{-}$and $\mathcal{A}^{+}$are extendable into weak arrangements of pseudo-line and, according to Lemma 9 have a smaller cardinality than $\mathcal{A}$ but include at least two arcs. By induction, they have at least 3 extremal points. Among these, there is one which is an extremal point of $\mathcal{A}$ belonging to the interior of $\mathcal{R}^{-}$(resp. $\mathcal{R}^{+}$). Notice that these extremal points do not belong to a same arc : otherwise, this arc would intersect $A$ twice. Hence, as the contact system $\mathcal{A}$ has an arc with two extremal points on it, it has at least 3 extremal points, what leads to a contradiction.

Hence, the lemma holds for any maximal contact system with at most $k$ arcs having two extremal points belonging to a same arc.

- Assume $\mathcal{A}$ does not include two extremal points belonging to a same arc. From the same construction and according to Lemma 8 the contact system $\mathcal{A}^{0}$ is maximal and, according to Lemma 9 it has cardinality at most $k$ and has two extremal points belonging to a same arc. According to the previous case, it has at least 3 extremal points. Among these, one is an extremal point of $\mathcal{A}$ belonging to the interior of $\mathcal{R}^{0}$. Together with the 2 extremal points of $\mathcal{A}$ belonging to the interior of $\mathcal{R}^{-}$and $\mathcal{R}^{+}$(as in the previous case), we get 3 extremal points, which lead to a contradiction. 
Lemma 11. Any contact system $\mathcal{A}$ extendable into a weak arrangement of pseudo-lines is extendable into a maximal contact system, each subsystem of which has at least 3 extremal points unless it has cardinality at most one.

Proof. As any subsystem $\mathcal{A}^{\prime}$ of the extension of $\mathcal{A}$ into a maximal contact system is extendable into a weak arrangement of pseudo-lines, the result follows from Lemma 10.

\subsection{Stretching}

Consider a maximal contact system of Jordan arcs $\mathcal{A}$, and the plane graph $G$ whose vertices are the points which are extremity of a least an arc of $\mathcal{A}$ and whose edges are the sub-arcs between such points.

Let $S$ be the set of the vertices of $G$ corresponding to points which are interior to no arc in $\mathcal{A}$.

Let $f$ be a function mapping $S$ to the vertices of a convex polygon, in compatible order.

Let $\alpha: V(G) \times V(G) \rightarrow[0,1]$ be a function such that:

$-\alpha(x, y)=0$ if and only if $y$ is interior to no arc in $\mathcal{A}$ or $x$ and $y$ are non adjacent in $G$.

$-\alpha\left(x_{1}, y\right)+\alpha\left(x_{2}, y\right)=1$ if $y$ is interior to some arc $A \in \mathcal{A}$ and has $x_{1}$ and $x_{2}$ as neighbors in $A$.

Then, $\Sigma=(S, f, \alpha)$ is a barycentric system on $G$.

Notation 1. Given an arc $A \in \mathcal{A}$, the path $P(A)$ is the path of $G$, whose vertices and edges are induced by $A$. The set $\mathcal{P}$ of these paths induces a covering of $G$ by edge disjoint paths.

By extension, given a contact system $\mathcal{A}^{\prime}, P\left(\mathcal{A}^{\prime}\right)$ denotes the union of the paths $P(A)\left(A \in \mathcal{A}^{\prime}\right)$.

Notation 2. Let $X \subseteq V(G)$. We note $\mathcal{A}(X)$ the set of the arcs of $\mathcal{A}$ having at least two points corresponding to vertices in $X$.

We now may link Theorem 3 with the stretching problem, using the next notations and lemmas.

Lemma 12. Let $\mathcal{A}^{\prime}$ be a subsystem of $\mathcal{A}$. Then $S\left(V\left(P\left(\mathcal{A}^{\prime}\right)\right)\right)=\delta\left(\mathcal{A}^{\prime}\right)$.

Proof. A vertex of $X=V\left(P\left(\mathcal{A}^{\prime}\right)\right)$ belongs to the $S(X)$ if and only if it has no incoming edge in $D_{\Sigma}$ (hence is an extremal point of $\mathcal{A}$ ) or if its has an incoming edge in $D_{\Sigma}$ from a vertex which does not belong to $X$ (and thus is internal to some arc in $\mathcal{A} \backslash \mathcal{A}^{\prime}$ or, equivalently, is an extremal point of $\mathcal{A}^{\prime}$ but not an extremal point of $\mathcal{A}$ ). Thus, $S(X)$ is the set of the extremal vertices of $\mathcal{A}^{\prime}$. 
Lemma 13. Let $X \subseteq V(G)$ have cardinality at least 2 and induce a connected subgraph of $G$. Then,

$$
\begin{aligned}
X & \subseteq V(P(\mathcal{A}(X))) \\
|S(X)| & \geq|S(V(P(\mathcal{A}(X))))|
\end{aligned}
$$

Proof. Inclusion (11) is straightforward as each edge of $G_{X}$ is included in the path corresponding to some arc of $\mathcal{A}(X)$.

Consider the connected graph $H$ obtained from $G_{X}$ by adding $k \geq 0$ paths corresponding to arcs in $\mathcal{A}(X)$ and assume that $|S(V(H))|=|S(X)|$ holds. Let $A$ be some arc of $\mathcal{A}(X)$, such that $P(A)$ does not belong to $H$.

- Assume $H$ include two vertices $v$ and $w$ of $P(A)$ but none of the edges of $P$ linking them.

Then, by adding those missing edges and vertices, no vertex is added to $S(V(H))$.

After this first completion, the internal vertices of $P(A)$ have at most one incoming edge from the complement of $H$.

- Otherwise, $H$ includes an interior vertex $v$ of $P(A)$, but none of the vertices between $v$ and an extremity $w$ of $P$.

Then, $v$ belongs to $S(V(H))$. By adding the sub-path from $v$ to $w$, exactly one vertex is added to the $S(V(H))$, namely $w$. As $P(A)$ has at least two vertices in common with $H, v$ is an interior vertex of $P(A)$. Moreover, as the previous case does not apply, $v$ had initially one incoming edge (exactly) from the complement of $H^{\prime}$. Thus, after the addition of the sub-path from $v$ to $w$, the vertex $v$ will no longer belong to $S(V(H))$.

Thus, we may add a new path to $H$ without increasing $|S(V(H))|$, which completes the induction.

Theorem 4. Let $\mathcal{A}$ be a contact system of Jordan arcs. Then, the following conditions are equivalent:

$\left(C_{1}\right) \mathcal{A}$ is stretchable,

$\left(C_{2}\right) \mathcal{A}$ is extendable into a weak arrangement of pseudo-lines,

Proof. It is straightforward that Condition $\left(C_{1}\right)$ implies condition $\left(C_{2}\right)$. According to Lemma 11, this second condition implies that $\mathcal{A}$ is extendable into a maximal contact system, each subsystem of which has at least 3 extremal points or has cardinality at most one. Consider any subset $X$ of vertices of $G$ with cardinality at least 2 and inducing a connected subgraph of $G$. Then, according to Lemma 13 and Lemma 12, $|S(X)| \geq|S(V(P(\mathcal{A}(X))))|=|\delta(\mathcal{A}(X))|$. But, either $\mathcal{A}(X)$ includes a single arc and hence $G_{X}$ is a path and $|S(X)| \geq 2$ or $\mathcal{A}(X)$ includes at least two arcs and then $|S(X)| \geq 3$, according to the hypothesis. The result now follows from the application of Theorem 3 . 


\section{Conclusion}

The equivalence between stretchability and extendibility into an arrangement of pseudo-lines for contact systems of Jordan arcs does not extend to intersection systems (stretchability of pseudoline arrangements is NP-hard, as proved by Short [5]). With more work, another equivalent condition may be given : A contact system is stretchable if and only if any subsystem has at least 3 extremal points on its unbounded region, unless it has at most an arc.

\section{References}

1. E. Colin de Verdière, M. Pocchiola, and G. Vegter, Tutte's barycenter method applied to isotopies, Proc. 13th Canad. Conf. Comput. Geom., 2001.

2. I. Fáry, On straight line representation of planar graphs, Acta Scientiarum Mathematicarum (Szeged) II (1948), 229-233.

3. M. Linial, L. Lovász, and A. Wigdersib, Rubber bands, convex embeddings and graph connectivity, Combinatorica 8 (1988), no. 1, 91-102.

4. D. Orden, Two problems in geometric combinatorics: Efficient triangulations of the hypercube;plane graphs and rigidity., Ph.D. thesis, University of Santander, Spain, 2003.

5. P. W. Shor, Stretchability of pseudolines is NP-hard, DIMACS Series in Discrete Mathematics and Theoretical Computer Science 4 (1991), 531-554.

6. S.K. Stein, Convex maps, Proc. Amer. Math. Soc., vol. 2, 1951, pp. 464-466.

7. E. Steinitz and H. Rademacher, Vorlesung über die Theorie der Polyeder, Springer, Berlin, 1934.

8. C. Thomassen, Planarity and duality of finite and infinite planar graphs, J. Combinatorial Theory, Series B 29 (1980), 244-271.

9. W.T. Tutte, Convex representations of graphs, Proc. London Math. Society, vol. 10, 1960, pp. 304-320.

10. How to draw a graph, Proc. London Math. Society, vol. 13, 1963, pp. 743768.

11. K. Wagner, Über eine Eigenschaft der Ebenen Komplexe, Math. Ann. 114 (1937), 570-590. 